\title{
A Parametric Study of Ridge-cut Explosive Bolts using Hydrocodes
}

\author{
Juho Lee* and Jae-Hung Han** \\ Department of Aerospace Engineering, KAIST, Korea Advanced Institute of Science and Technology, Daejeon 305-701, Republic \\ of Korea
}

\author{
YeungJo Lee L*** $^{* *}$ \\ The 4th R\&D Institute, Agency for Defense Development, Daejeon 305-600, Republic of Korea
}

\section{Hyoungjin Lee****}

PGM R\&D Lab., LIG NEX1 Co. Ltd., Seongnam 463-400, Republic of Korea

\begin{abstract}
Explosive bolts are one of pyrotechnic release devices, which are highly reliable and efficient for a built-in release. Among them, ridge-cut explosive bolts which utilize shock wave generated by detonation to separate bolt body produce minimal fragments, little swelling and clean breaks. In this study, separation phenomena of ridge-cut explosive bolts or ridge-cut mechanism are computationally analyzed using Hydrocodes. To analyze separation mechanism of ridge-cut explosive bolts, fluid-structure interactions with complex material modeling are essential. For modeling of high explosives (RDX and PETN), Euler elements with Jones-Wilkins-Lee E.O.S. are utilized. For Lagrange elements of bolt body structures, shock E.O.S., Johnson-Cook strength model, and principal stress failure criteria are used. From the computational analysis of the author's explosive bolt model, computational analysis framework is verified and perfected with tuned failure criteria. Practical design improvements are also suggested based on a parametric study. Some design parameters, such as explosive weights, ridge angle, and ridge position, are chosen that might affect the separation reliability; and analysis is carried out for several designs. The results of this study provide useful information to avoid unnecessary separation experiments related with design parameters.
\end{abstract}

Key words: Pyrotechnics, Ridge-Cut Explosive Bolts, Hydrocodes, Separation Behavior Analysis.

\section{Introduction}

Explosive bolts are reliable and efficient pyrotechnic release devices that can be used for diverse applications including launcher operation, stage separation, release of external tanks, and thrust termination $[1,2]$. The explosive bolt, which has a form of a bolt body with the cavity filled with a removable cartridge or an explosive charge, is more reliable and robust than other types of release devices, due to its simplicity. Most of the explosive bolts can be categorized as high-explosive type and pressure type, based on their separation mechanism. Ridge-cut explosive bolts, which are one of the high-explosive types, utilize shock wave generated by detonation to separate bolt bodies. Furthermore, it is well known that the ridge-cut explosive bolt produces minimal shrapnel, little swelling, and a clean break [3].

While the usage of explosive bolts is quite prevalent, designing procedures still rely on heritage with repetitive experiments. To overcome this limitation, the computational analysis method for the explosive bolts is needed. Based on the computational analysis, separation reliability of the explosive bolts can be evaluated without time-consuming and costly experiments. Furthermore, complex separation phenomena and characteristics can be studied. In many
This is an Open Access article distributed under the terms of the Creative Commons Attribution Non-Commercial License (http://creativecommons.org/licenses/by$\mathrm{nc} / 3.0 /$ ) which permits unrestricted non-commercial use, distribution, and reproduction in any medium, provided the original work is properly cited. (c)

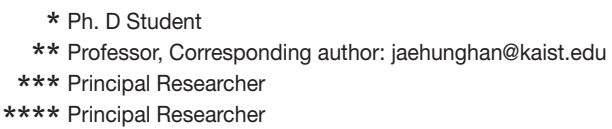


cases, complex separation characteristics cannot be fully understood based on simple theories and experimental results. Based on the computational method regarding the blast loading on structures [4-10], the authors proposed the computational analysis method for the separation behaviors of the ridge-cut explosive bolts [11]. Previous study clearly showed the separation mechanism of the ridge-cut explosive bolts from the computation analysis, and the computation scheme was verified by comparing with the experimental results. From the separation characteristics study regarding the confinement conditions, design improvements were proposed [11]. However, previous study focused only on the specific confinement condition. To widely apply the computation analysis method for the designing procedures, it still requires further studies by applying computation analysis method to general design parameters of explosive bolts.

In this study, computational analysis methodology for ridge-cut explosive bolts separation behavior is revised and improved in some aspects. By utilizing this numerical analysis framework, a parametric study with design parameters of ridge-cut explosive bolts is performed, and meaningful analysis results are obtained to apply design procedures.

\section{Separation mechanism of ridge-cut explo- sive bolts}

In this study, the ridge-cut explosive bolts as depicted in Fig. 1, which are designed by the author's previous experience $[12,13]$, are computationally analyzed to understand the separation characteristics. The ridge-cut explosive bolts are designed based on the theory of ridge-cut mechanism [11].
A 17-4PH stainless steel is used for the bolt body and the fixtures of the ridge-cut explosive bolts. A removable initiator and the priming material (Lead Azide, LA) are utilized to initiate the high explosives. For the high explosives, PETN and RDX are utilized. The separation reliability of the ridge-cut explosive bolts is verified by repetitive separation experiments. The separation plane observed by separation experiments is shown in Fig. 1. However, the pyroshock generated by the exploding bolts exceeded 10,000 g above $4,000 \mathrm{~Hz}$ at near field. If the explosive weight can be reduced without compromising the separation reliability, the probability of malfunctions in nearby electrical components can be significantly reduced.

Unlike other types of explosive bolts, the separation mechanism of ridge-cut explosive bolts cannot be understood by classical fracture mechanics, but it can be explained by ridge-cut mechanism (Fig. 2). Ridge-cut technique or mechanism can be explainable by the fracture mechanism, when a metal structure has ridge on one side and high explosives detonate on the other side. At first, shock waves generated by detonation of high explosives are emitted

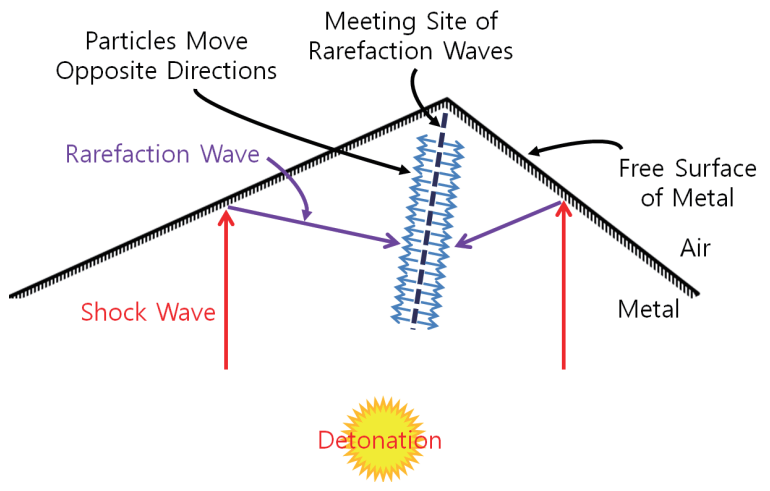

Fig. 2. Ridge-cut mechanism

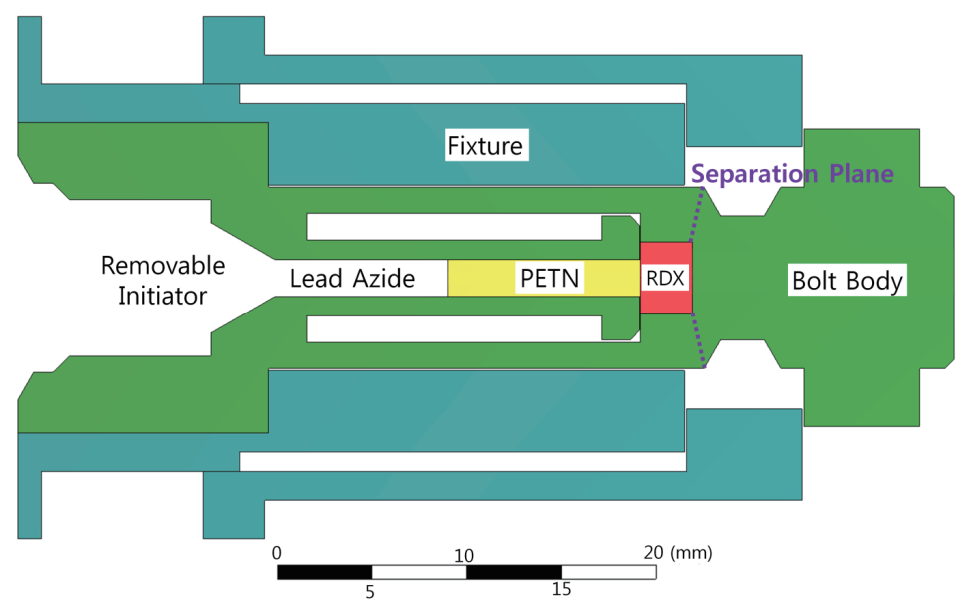

Fig. 1. Cross-sectional diagram of ridge-cut explosive bolts 
into the metal structure. When the shock waves encounter the free surface of metal structure, they reflect back as the rarefaction waves (or release waves). When the rarefaction waves collide at the meeting site of rarefaction waves, particles at the meeting site move in opposite directions. These movements create high tensile stress at the meeting site, resulting in the separation.

\section{Improvements of computational analysis methodology}

Ridge-cut explosive bolts and their separation mechanism can be computationally analyzed in AUTODYN (one of commercial Hydrocodes), due to necessity of Euler-Lagrange interactions and complex material models. Computational analysis of ridge-cut explosive bolts are performed in 2-D axisymmetric to minimize computational costs. The computational analysis method for the ridge-cut explosive bolts was well developed in previous study [11]. The analysis method is summarized and the improvements are introduced here. The improvements include the structural boundary condition and the parameter values of the Shock E.O.S. (the Gruneisen constant and the $U-u$ Hugoniot). The estimation method for the material properties of the high explosives with slightly different density is also proposed.

\subsection{Summary of computational analysis method}

Due to geometric complexity of ridge-cut explosive bolts, geometric modeling including structure meshing, the connections and body interaction are performed in ANSYS Workbench Explicit Dynamics. The structural mesh is constructed via a quadrilateral dominant method with Lagrange elements $0.2 \mathrm{~mm}$ in size, as shown in Fig. 3. Smaller element sizes generally induce accurate analysis results, but they require high computational costs. Therefore, optimal mesh size is determined to guarantee converged numerical results and fast computation time. In this study, the connections between the bolt body and the fixtures are modeled as mesh connections, allowing the joining of the meshes of topologically disconnected bodies. In ANSYS AUTODYN, the mesh connections are recognized as bonded connections.

The remaining modeling steps, including Eulerian modeling and materials modeling, are performed in ANSYS AUTODYN. Materials modeling, definition of initial conditions, Euler parts, Euler boundary conditions, structural boundary condition, Euler-Lagrange interaction, detonation, and solution controls are necessary in AUTODYN. In the ridge-cut explosive bolts, the removable initiator and the priming material are utilized merely to initiate detonation of PETN and RDX. Therefore, initiator and LA that generate negligible blast loading on bolt body are neglected in computational analysis. For high explosives, RDX and PETN, Jones-Wilkins-Lee (JWL) E.O.S. are used. For 17-4PH stainless steel (material of the bolt body and the fixtures), the ShockE.O.S., the Johnson-Cook strength model, and the principal stress failure model are used. Surrounding air is defined as the ideal gas with material properties at 1 atmosphere.

Initial density and internal energy of each material are defined as initial conditions. The air, PETN, and RDX are constructed as the Euler parts. The size of Euler elements for air and high explosives is $0.1 \mathrm{~mm}$, which is half of Lagrange elements (Fig. 4). To model the spread-out of detonation products, flow out Euler boundary condition is applied to

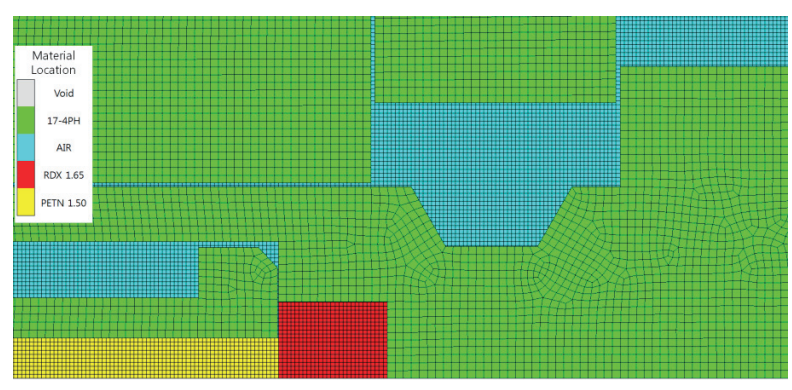

Fig. 4. Elements size comparison between Lagrange and Euler.

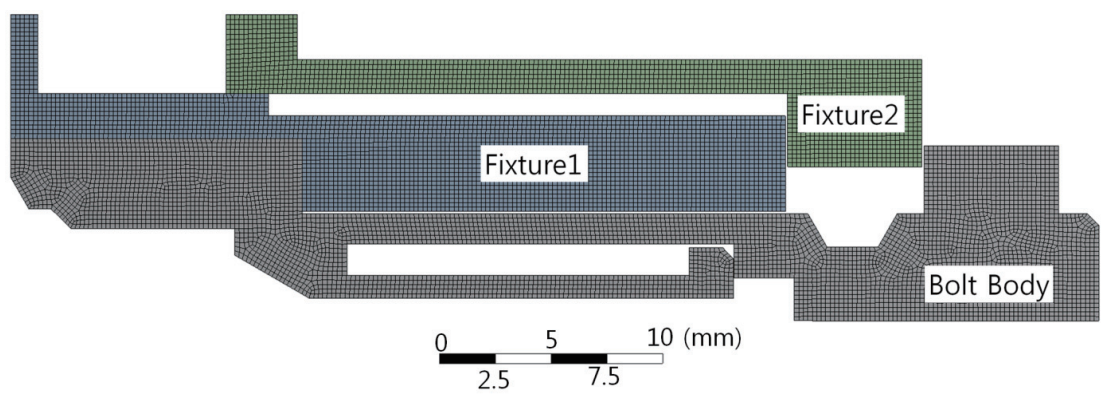

Fig. 3. Structure geometric modeling and meshing of the ridge-cut explosive bolts. 
the boundary of the Euler parts, excluding the axisymmetric axis. In this study, a transmit boundary condition is utilized as a structural boundary condition, as shown in Fig. 5. The transmit boundary condition ensures the stress waves to transmit out without reflection at the boundary. It is modeled as infinite structures are attached at the boundary with identical impedance value (the density multiplied by the sound velocity). It is defined that detonation is initiated at the left bottom point of PETN in 2-D model. A single time step is determined by CFL (Courant-Friedrichs-Lewy) condition which guarantees stability and accuracy of the solution. The computational analysis is performed up to 0.01 ms.

\subsection{Modeling of high explosives}

For modeling of detonation products from high explosives, the Jones-Wilkins-Lee (JWL) E.O.S. [14] are widely used. It can evaluate experimental geometries of detonation products from initiation to large expansions.

$$
P=A\left(1-\frac{\omega}{R_{1} V}\right) e^{-R_{1} V}+B\left(1-\frac{\omega}{R_{2} V}\right) e^{-R_{2} V}+\frac{\omega E}{V}
$$

Here, $V$ is the relative volume $v / v_{0} ; E$ is the internal energy; and $A, B, R_{1}, R_{2}, \omega$ are empirical parameters determined by detonation experiments. The detonation velocity and information regarding the Chapman-Jouguet (C-J) point as the reference state of JWL E.O.S. are also required. The C-J point is the position where the reaction (or detonation) is complete.

Material properties of RDX and PETN for computational analysis are given in Table 1 . The density of RDX and PETN for reference model is $1.65 \mathrm{~g} / \mathrm{cm}^{3}$ and $1.50 \mathrm{~g} / \mathrm{cm}^{3}$, respectively. Material properties of COMP A-3 and PETN 1.50 supplied by AUTODYN are utilized for RDX 1.65 and PETN 1.50.

In this study, the material properties of high explosive for different explosive density are needed. The estimation method is derived for the material properties with slightly different density of up to $10 \%$. The linear dependence of detonation velocity $D$ upon initial density $\rho_{0}$ can be used to estimate the detonation velocity. For most explosives, the following relationships are quite accurate for a broad range of density [15]:

$$
D=j+k \rho_{0}
$$

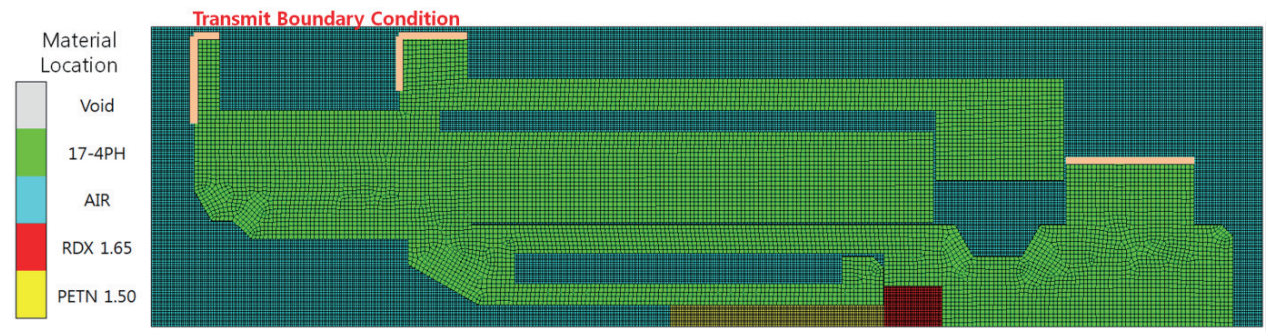

Fig. 5. Euler parts including air, RDX, PETN and transmit boundary condition.

\begin{tabular}{|c|c|c|c|c|c|c|c|}
\hline Parameter of JWL E.O.S. & & PETN 1.77 & PETN 1.50 & PETN 1.26 & RDX 1.815 & RDX 1.65 & RDX 1.485 \\
\hline Density $\rho_{0}$ & $\left(\mathrm{~g} / \mathrm{cm}^{3}\right)$ & 1.77 & 1.50 & 1.26 & 1.815 & 1.65 & 1.485 \\
\hline Parameter $A$ & $(\mathrm{kPa})$ & $6.1705 \times 10^{8}$ & $6.253 \times 10^{8}$ & $5.731 \times 10^{8}$ & $7.826 \times 10^{8}$ & $6.113 \times 10^{8}$ & $4.634 \times 10^{8}$ \\
\hline Parameter $B$ & $(\mathrm{kPa})$ & $1.6926 \times 10^{7}$ & $2.329 \times 10^{7}$ & $2.016 \times 10^{7}$ & $1.335 \times 10^{7}$ & $1.065 \times 10^{7}$ & $8.049 \times 10^{6}$ \\
\hline Parameter $R_{1}$ & (none) & 4.4 & 5.25 & 6.0 & 4.4 & 4.4 & 4.4 \\
\hline Parameter $R_{2}$ & (none) & 1.2 & 1.6 & 1.8 & 1.2 & 1.2 & 1.2 \\
\hline Parameter $\omega$ & (none) & 0.25 & 0.28 & 0.28 & 0.32 & 0.32 & 0.32 \\
\hline Detonation velocity $D$ & $(\mathrm{~m} / \mathrm{s})$ & $8.30 \times 10^{3}$ & $7.45 \times 10^{3}$ & $6.54 \times 10^{3}$ & $8.858 \times 10^{3}$ & $8.3 \times 10^{3}$ & $7.713 \times 10^{3}$ \\
\hline $\begin{array}{l}\text { C-J Energy per unit } \\
\text { volume } E_{0}\end{array}$ & $\left(\mathrm{~kJ} / \mathrm{m}^{3}\right)$ & $1.01 \times 10^{7}$ & $8.56 \times 10^{6}$ & $7.19 \times 10^{6}$ & $9.79 \times 10^{6}$ & $8.9 \times 10^{6}$ & $8.01 \times 10^{6}$ \\
\hline C-J Pressure & $(\mathrm{kPa})$ & $3.35 \times 10^{7}$ & $2.2 \times 10^{7}$ & $1.4 \times 10^{7}$ & $3.718 \times 10^{7}$ & $3 \times 10^{7}$ & $2.359 \times 10^{7}$ \\
\hline
\end{tabular}

Table 1. Revised Material Properties of High Explosive with different density 
For RDX, $j$ is 2.56 and $k$ is 3.47. For PETN, $j$ is 1.82 and $k$ is 3.7 when the density is between $0.37 \mathrm{~g} / \mathrm{cm}^{3}$ and $1.65 \mathrm{~g} / \mathrm{cm}^{3}$.

Also, accurate estimation of the C-J pressure from the detonation velocity and the initial density is as follows [15]:

$$
P_{C J}=\rho_{0} D^{2}\left(1-0.7215 \rho_{0}^{0.04}\right)
$$

It is not unreasonable to assume that the C-J energy per unit volume $E_{0}$ is proportional to density [14]. Within $10 \%$ variation of density, parameters $R_{1}, R_{1}, \omega$ can be retained [14]. To calculate parameters $A$ and $B$, following system of equations should be solved [14]. Even though parameter $C$ will be calculated, it is not necessary for our Hydrocodes modeling.

$$
\begin{aligned}
& P_{C J}=A e^{-R_{1} V_{C J}}+B e^{-R_{2} V_{C J}}+C \frac{1}{V_{C J}^{\omega+1}} \\
& E_{0}-\frac{1}{2} P_{C J}\left(1-V_{C J}\right)=A \frac{e^{-R_{1} V_{C J}}}{R_{1}}+B \frac{e^{-R_{2} V_{C J}}}{R_{2}}+C \frac{1}{\omega V_{C J}^{\omega}} \\
& \frac{P_{C J}}{1-V_{C J}}=A R_{1} e^{-R_{1} V_{C J}}+B R_{2} e^{-R_{2} V_{C J}}+C(\omega+1) \frac{1}{V_{C J}^{(\omega+2)}}
\end{aligned}
$$

Here, $V_{C J}$ is the relative volume $v / v_{0}$ at C-J point:

$$
V_{C J}=\left(1-\frac{P_{C J}}{\rho D^{2}}\right)
$$

Obviously, if we apply this calculation for reference density, for which the parameters of JWL E.O.S. are known, the calculated parameters $A$ and $B$ are exactly the same as the known values.

For the material properties of PETN with different explosive densities, material properties of PETN 1.26 and PETN 1.77 are utilized, which is supplied by AUTODYN. However, the material properties of RDX with different explosive densities are not available. Therefore, the material properties of RDX with $10 \%$ variations in density are estimated. Material properties of high explosives with different explosive densities are also summarized in Table 1.

\subsection{Modeling of 17-4PH stainless steel}

For 17-4PH stainless steel, the Shock E.O.S. are utilized, which is the Mie-Gruneisen form of E.O.S. [16] that uses the shock Hugoniot as reference.

$$
P-P_{H}=\frac{\gamma}{v}\left(e-e_{H}\right)
$$

Here, $P$ is the pressure, $\gamma$ is the Gruneisen constant, $v$ is the specific volume, and $e$ is the specific internal energy.
Subscript $H$ denotes the shock Hugoniot, which is defined as the locus of all shocked states for each material. Here, we need the $P-v$ Hugoniot for the Shock E.O.S.. This Hugoniot can be obtained from the $U-u$ Hugoniot or the relationship between shock and particle velocities.

$$
U=C_{0}+s u
$$

Here, $C_{0}$ and $s$ are empirical parameters obtained from the least-square fits to the experimental results $[17,18]$. For the shock E.O.S. of 17-4PH stainless steel, the density, the specific heat, the Gruneisen constant, and the $U$ - $u$ Hugoniot (empirical parameters $C_{0}$ and $s$ ) are necessary. Only the density and the specific heat are known [19].

In this study, the Gruneisen constant is theoretically estimated [16] from the volumetric thermal expansion coefficient, the specific heat $C_{v}$, and the bulk modulus:

$$
\gamma=-\frac{1}{\rho_{0} C_{v}}\left(\frac{\partial P}{\partial\left(V / V_{0}\right)}\right)\left(\frac{\partial\left(V / V_{0}\right)}{\partial T}\right)_{\substack{P=0 \\ T=298 K}}
$$

The volumetric thermal expansion coefficient is three times of the linear thermal expansion coefficient. All the parameters for the theoretical calculation are known [19].

Because the $U-u$ Hugoniot of $17-4 \mathrm{PH}$ stainless steel is unknown, the relationship pertaining to $\mathrm{PH} 13-8 \mathrm{MO}$ stainless steel [20], which is the material most similar in terms of this relationship, is utilized in this study. PH13-8MO stainless steel is also a precipitation-hardening martensitic stainless steel, like 17-4PH stainless steel.

For modeling of the high-strain-rate plastic deformation or flow stress, Johnson-Cook strength model is used. The Johnson-Cook equation [21] is an empirical constitutive equation regarding the plastic deformation of metals with large strains, high strain rates, and high temperatures.

$$
\sigma=\left(\sigma_{0}+B \varepsilon^{n}\right)\left(1+C \ln \frac{\dot{\varepsilon}}{\dot{\varepsilon}_{0}}\right)\left[1-\left(\frac{T-T_{r}}{T_{m}-T_{r}}\right)^{m}\right]
$$

Here, $\sigma$ is the yield stress or flow stress, $\sigma_{0}$ is the static yield stress, $B$ is the hardening constant, $\varepsilon$ is the strain, $n$ is the hardening exponent, $C$ is the strain rate constant, $\dot{\varepsilon}$ is the strain rate, $\dot{\varepsilon}_{0}$ is the reference strain rate, $T$ is the temperature, $T_{r}$ is the reference temperature, $T_{m}$ is the melting point, and $m$ is the thermal softening exponent.

For 17-4PH stainless steel (H1025, HRC38), only the static yield stress is known [19]. Other parameters are assumed by averaging those of 4340 steel and S-7 tool steel, which have the same density and the specific heat as 17-4PH stainless steel. In addition, the static yield stress and the Rockwell hardness of $17-4 \mathrm{PH}$ stainless steel are similar with the average values of 4340 steel and S-7 tool 
steel.

To model ridge-cut mechanism, the principal stress failure criteria are utilized. With the principal stress failure model, failure of each element is initiated if the maximum principal tensile stress exceeds a critical value of normal tensile stress. The appropriate failure criteria for the ridge-cut explosive bolts are determined from previous study [11]. Revised material properties of $17-4 \mathrm{PH}$ stainless steel for computational analysis are summarized in Table 2.

\section{Computational analysis results of ridge- cut explosive bolts}

By utilizing developed numerical analysis framework, separation behavior of the ridge-cut explosive bolts is computationally analyzed. At first, the principal stress failure model is not used to clearly observe propagation, reflection of shock waves, and collision of reflected rarefaction waves. Detonation is initiated at the left bottom point of PETN as analysis starts $(0 \mathrm{~ms})$. Detonation of PETN and RDX is finished at around 1.7 s (Fig. 6(a)). Shock waves from detonation are emitted into the bolt body and encounter the free surface near the ridge around $2.1 \mu \mathrm{s}$ (Fig. 6(b)). At the free surface, shock waves reflect back as rarefaction waves, and these reflected rarefaction waves collide at the meeting site of rarefaction waves at around $2.5 \mu$ s (Fig. 6(c)). From this computational analysis, the ridge-cut explosive bolts are expected to be separated at around $2.5 \mu \mathrm{s}$.

From the previous study [11], the appropriate principal stress failure criteria are determined as $2.8 \mathrm{GPa}$ critical value of normal tensile stress. For comparison, material statuses at $5.0 \mu$ s are given in Fig. 7, which use different critical values of normal tensile stress ranging from 2.4 GPa to $3.2 \mathrm{GPa}$. If low failure criteria are used, widespread failures are observable as shown in Fig. 7(a) and (b), and these are unlike the separation experiments. In the experiments, clean ridge breaks were observed. Furthermore, this makes the separation analysis results insensitive to parameter variations. If high failure criteria are applied, localized failures will be observed as shown in Fig. 7(d) and (e), which imply no separation of the explosive bolts. This makes it difficult to evaluate the separation reliability.

Table 2. Revised Material Properties of 17-4PH Stainless Steel

\begin{tabular}{l|l}
\hline \hline Parameter of Shock E.O.S. & $17-4 \mathrm{PH}$ Stainless Steel \\
\hline Density $\rho_{0}$ & $7.81 \mathrm{~g} / \mathrm{cm}^{3}$ \\
Gruneisen coefficient $\gamma$ & 1.36 \\
Empirical parameter $C_{0}$ & $4550 \mathrm{~m} / \mathrm{s}$ \\
Empirical parameter $s$ & 1.41 \\
Reference temperature $T_{r}$ & $300 \mathrm{~K}$ \\
Specific Heat $C_{v}$ & $460 \mathrm{~J} / \mathrm{kgK}$ \\
\hline \hline Parameter of Johnson Cook strength model & \\
\hline Shear modulus & $7.7437 \times 10^{7} \mathrm{kPa}$ \\
Static yield stress $\sigma_{0}$ & $1.172 \times 10^{6} \mathrm{kPa}$ \\
Hardening constant $B$ & $4.935 \times 10^{5} \mathrm{kPa}$ \\
Hardening exponent $n$ & 0.22 \\
Strain rate constant $C$ & 0.013 \\
Thermal softening exponent $m$ & 1.015 \\
Melting point $T_{m}$ & $1778 \mathrm{~K}$ \\
Reference strain rate $\dot{\varepsilon}_{0}$ & 1.0 \\
\hline \hline Parameter of principal stress failure model & \\
\hline Critical value of normal tensile stress $\sigma_{c}$ & $2.8 \times 10^{6} \mathrm{kPa}$ \\
\hline \hline Parameter of geometric strain erosion model & \\
\hline Erosion strain & 1.5 \\
Type of geometric strain & Instantaneous \\
\hline
\end{tabular}


From the computational analysis results of ridge-cut explosive bolts, separation reliability can be estimated. Number, position, direction, and density of failed Lagrangian elements can be considered for the evaluation. Through comparison with computational results of the reference ridge-cut explosive bolts, the separation reliability can be evaluated for diverse designs of ridge-cut explosive bolts.

\section{Parametric study of ridge-cut explosive bolts}

In this study, a separation-behavior analysis method is developed for ridge-cut explosive bolts, and this method allows evaluation of the separation reliability for various ridge-cut explosive bolt designs. Some design parameters are chosen that might affect the separation reliability, and behavior analysis is carried out for several designs.

\subsection{Explosive weight}

In the reference ridge-cut explosive bolts, $47 \mathrm{mg}$ of PETN and $50 \mathrm{mg}$ of RDX are used to break the bolt body. When the explosive weight is increased or decreased, shock propagation and the separation reliability are affected. In this study, to observe the effect of the explosive weight on the separation reliability, only the densities of explosives are varied, and the dimensions of the ridge and the fixture are unchanged. Here, four cases are considered as follows:

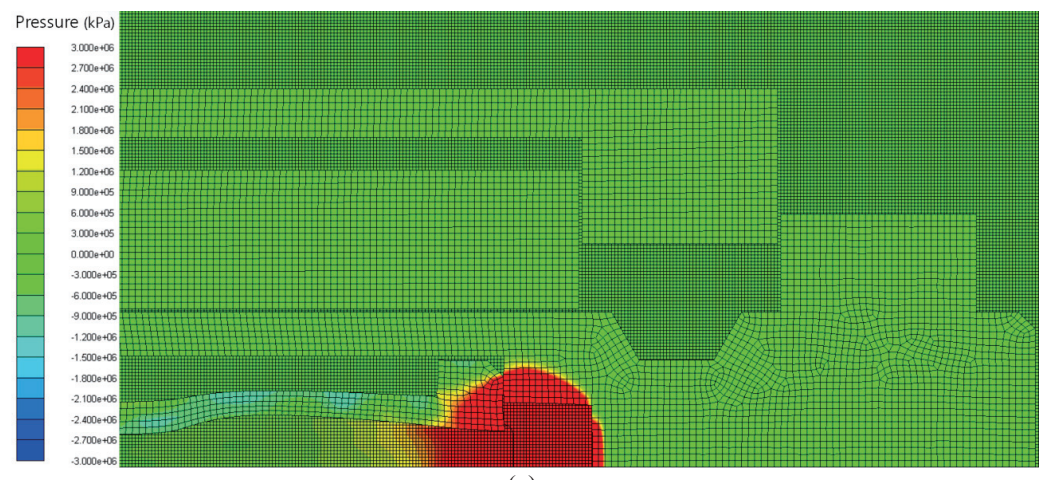

(a)

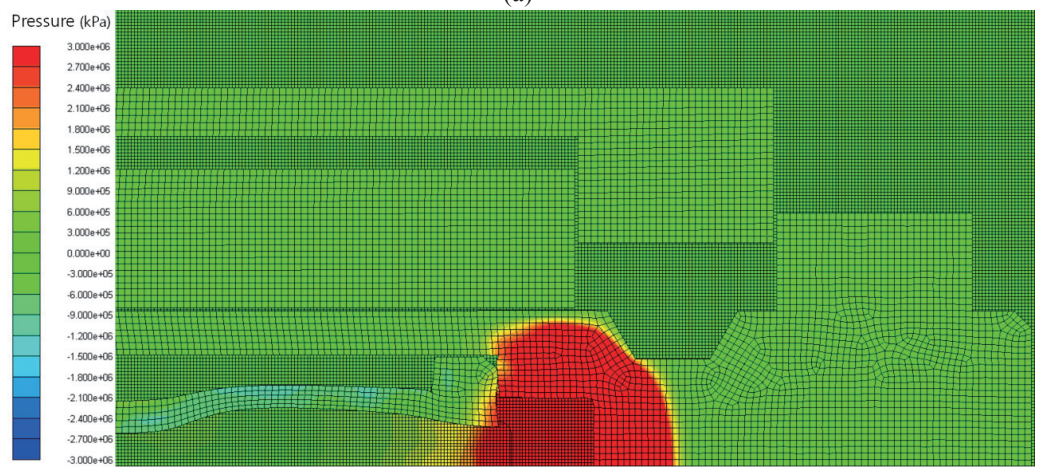

(b)

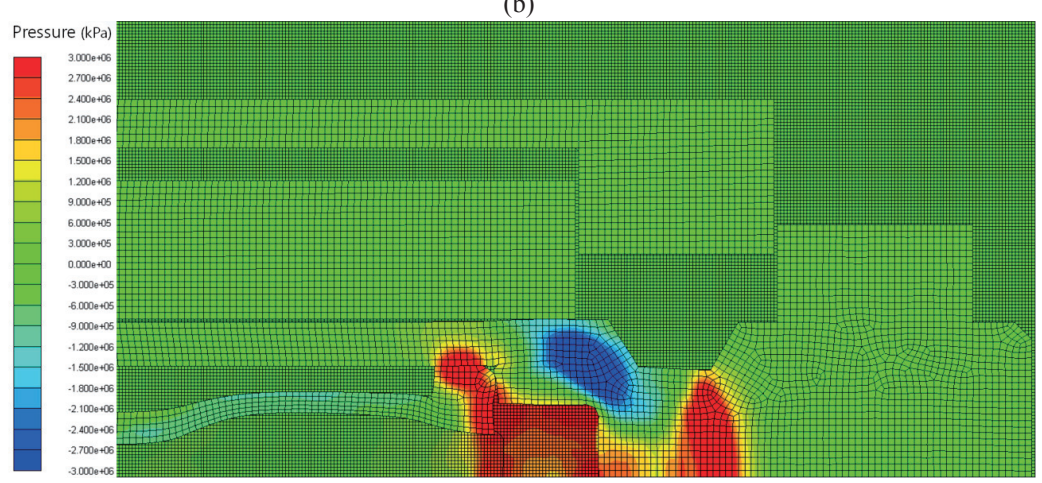

(c)

Fig. 6. Pressure contours without failure modeling: (a) at $1.7 \mu \mathrm{s} ;$ (b) at $2.1 \mu \mathrm{s} ;$ (c) at $2.5 \mu \mathrm{s}$. 


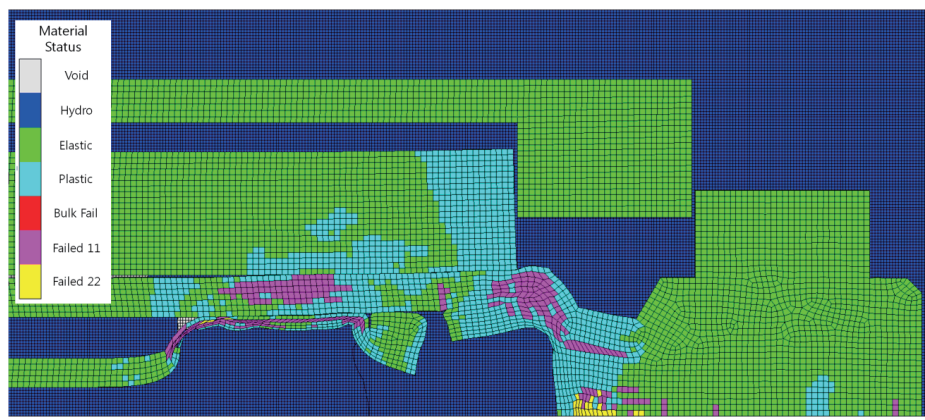

(a)

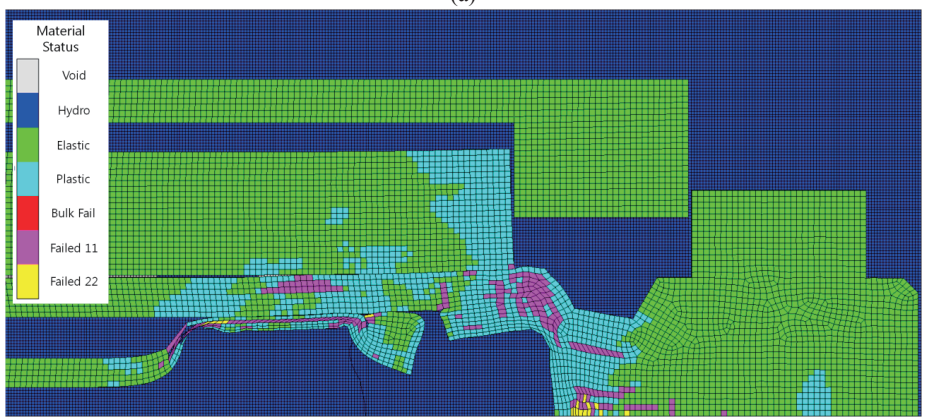

(b)

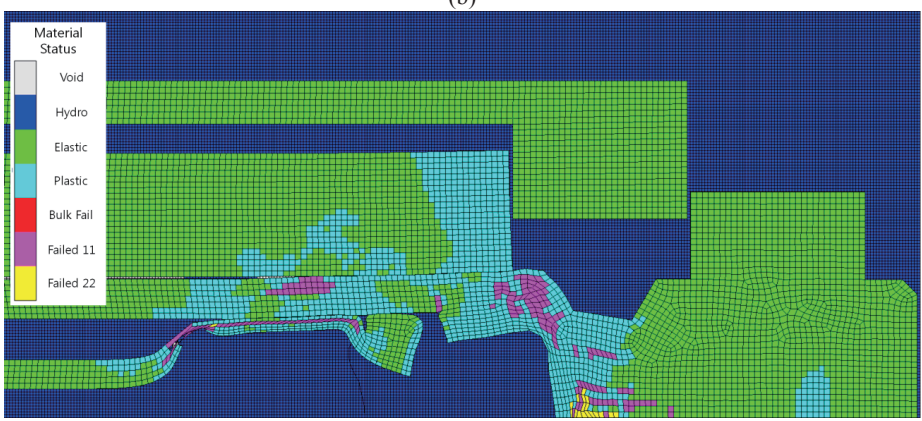

(c)

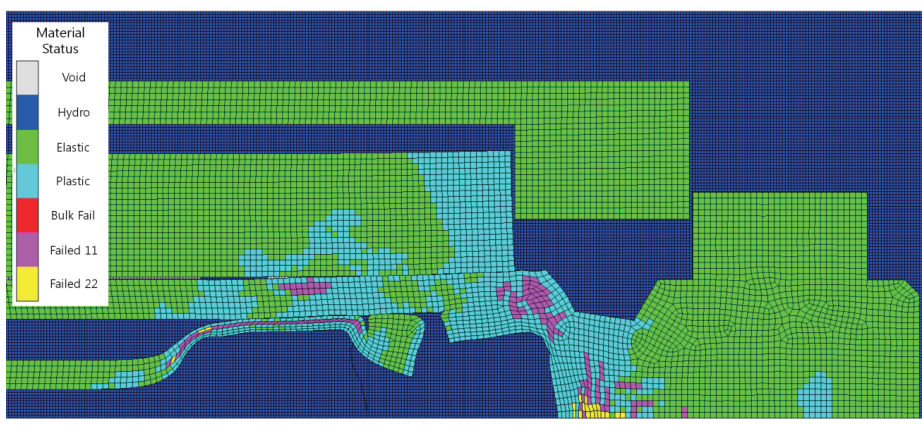

(d)

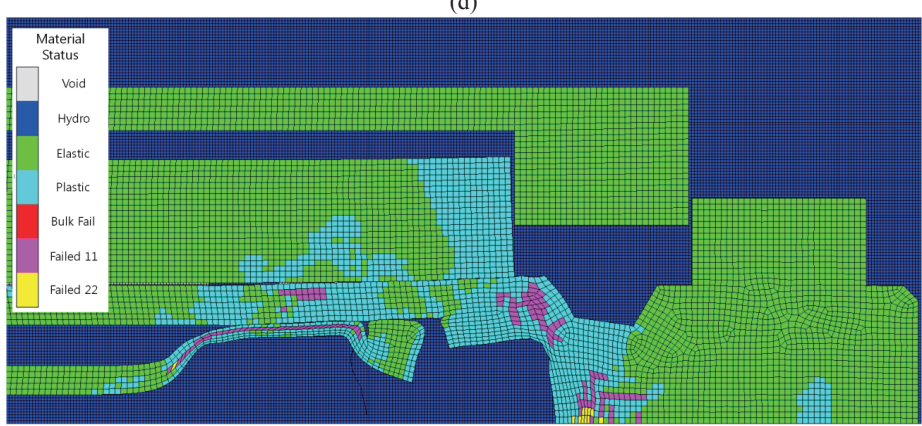

(e)

Fig. 7. Material statuses at $5.0 \mu$ s for different principal stress failure criteria: (a) $2.4 \mathrm{GPa}$; (b) $2.6 \mathrm{GPa}$; (c) $2.8 \mathrm{GPa}$; (d) $3.0 \mathrm{GPa}$; (e) $3.2 \mathrm{GPa}$. 
1) the explosive densities of RDX and PETN are increased (RDX 1.815 and PETN 1.77), 2) the explosive density of RDX is increased (RDX 1.815 and PETN 1.50), 3) the explosive densities of RDX and PETN are decreased (RDX 1.485 and PETN 1.26), and 4) the explosive density of PETN is decreased (RDX 1.65 and PETN 1.26).

To demonstrate the separation reliability, the material status at $5.0 \mu \mathrm{s}$ is shown in Fig. 8. According to the comparison with the results for the reference model (Fig. 7 (c)), it is easily inferred that the separation reliability increases as the explosive weight increases. However, this inference is not always correct, due to the side effects of excess explosive. Furthermore, it is quite clear that the separation reliability is affected by the explosive weight

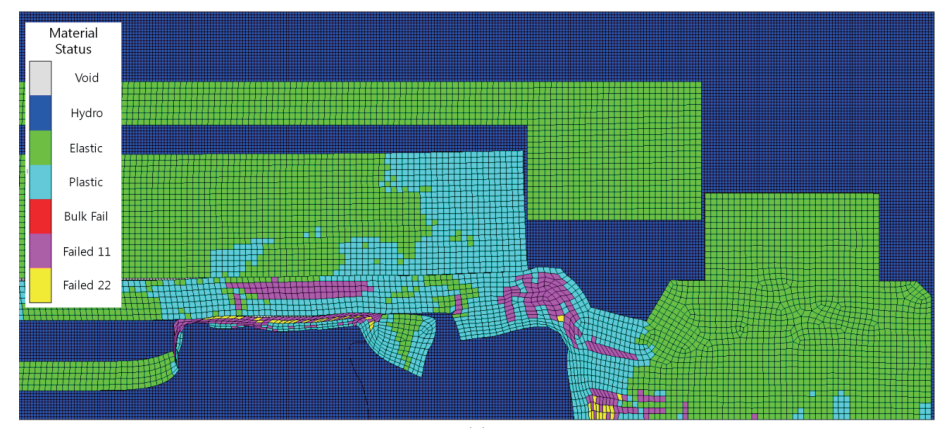

(a)

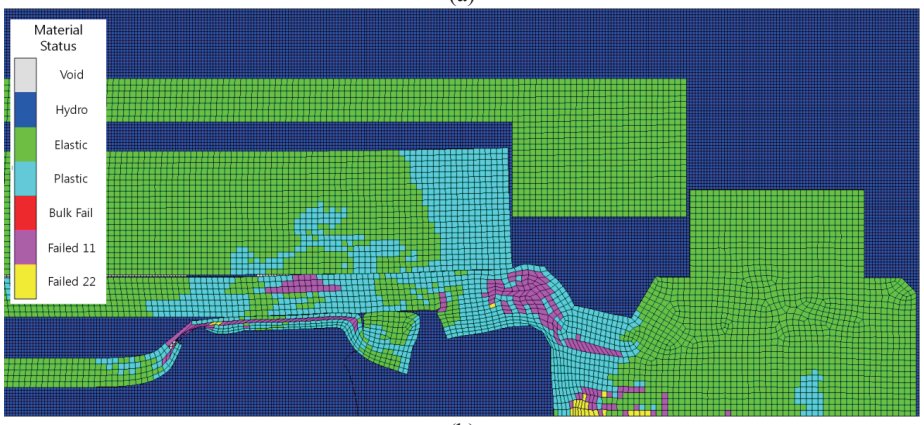

(b)

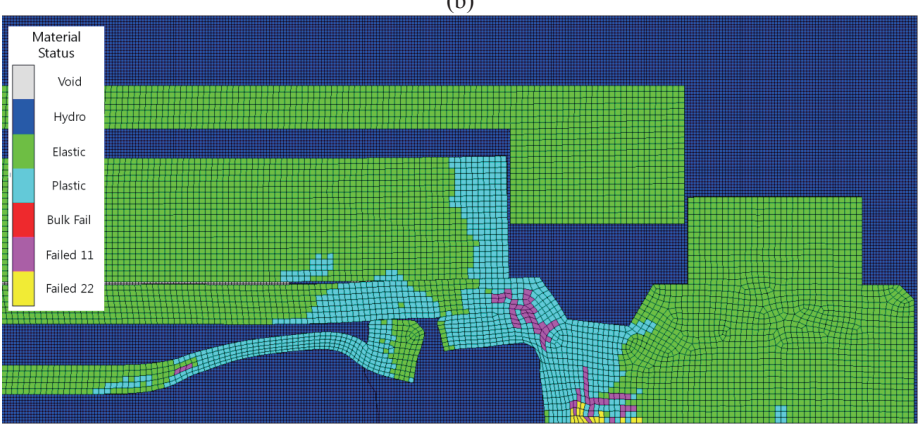

(c)

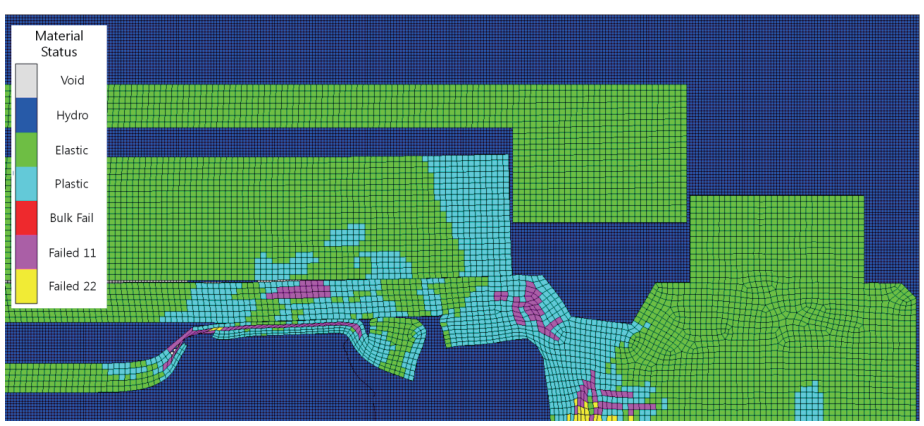

(d)

Fig. 8. Material statuses at $5.0 \mu$ s for different explosive weight (a) High density RDX and PETN; (b) High density RDX; (c) Low density RDX and PETN; (d) Low density RDX. 
of RDX, rather than the explosive weight of PETN. This is because PETN is utilized to initiate RDX, and the shock waves mainly generated by the RDX break the bolt body.

\subsection{Ridge angle}

For the reference ridge-cut explosive bolts, the ridge angle of the bolt body is $120^{\circ}$. When the ridge angle is increased or decreased, the reflection of shock waves, the superposition of release waves, and the separation reliability are affected. In this study, the ridge angle (which affects the reflection of shock waves) is increased and decreased by $10^{\circ}$. The meshes used for the increased and decreased ridge angle models are shown in Fig. 9(a) and Fig. 9(b), respectively, with a dashed outline of the reference model. The bisection line of the ridge is indicated by a dotted red line in the reference model and by a dashed black line in the models, with increased and decreased ridge angles. To determine the separation reliability, the material status at $5.0 \mu$ s is shown in Fig. 10. Even though the increased ridge-angle model has a larger volume around the ridge of the explosive bolt, it has higher separation reliability than either the decreased ridge angle model or the reference model. These phenomena can be explained by the rotation of the bisection line of the ridge. In the increased ridge-angle model, this line rotates counterclockwise with respect to the vertex of the ridge, which implies a length decrement of the line, as shown in Fig. 9(a). Therefore, fewer elements (i.e., a smaller portion of the bolt) must fail to induce bolt separation, which implies higher separation reliability for the same explosive weight. On the other hand, in the decreased ridge-angle model, the bisection line of the ridge rotates clockwise with respect to the vertex of the ridge, which implies a length increment of the line, as shown in Fig. 9(b). Therefore, more elements (i.e., a larger portion of the bolt) must fail to induce bolt separation, which implies lower separation reliability for the same explosive weight.

\subsection{Ridge position}

As with the other parameters, ridge position can be adjusted. When the ridge position is shifted to the left or right, the reflection of shock waves, the superposition of release waves, and the separation reliability are affected. In this study, the ridge position is shifted to the left and to the right by $5 \mathrm{~mm}$. To observe the effect of ridge position on the separation reliability, the dimensions of the ridge and the fixture are uniformly varied, as shown in Fig. 11(a) and Fig. 11(b). To illustrate the separation reliability, the material status at $5.0 \mu \mathrm{s}$ is shown in Fig. 12. Even though the left-shifted ridge-position model has a smaller volume around the ridge of the explosive bolt, it has lower separation reliability than either the rightshifted ridge-position model or the reference model. These phenomena can be explained by the cancellation of the propagating shock waves and reflected release

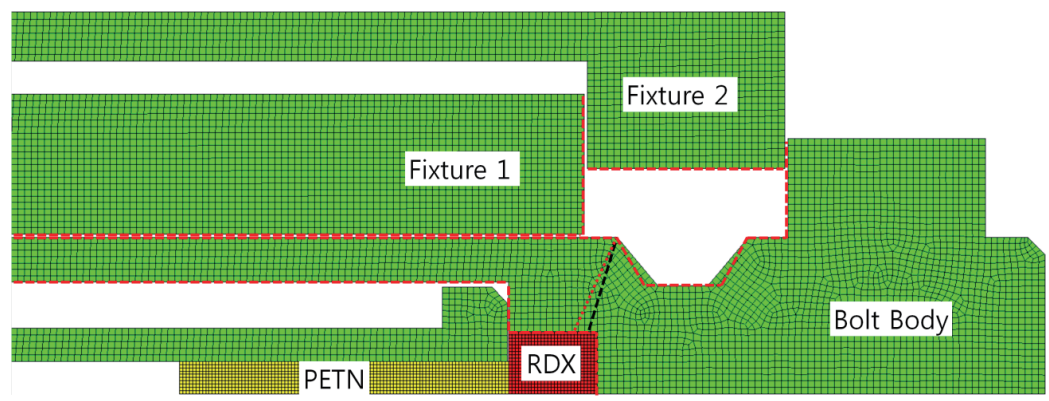

(a)

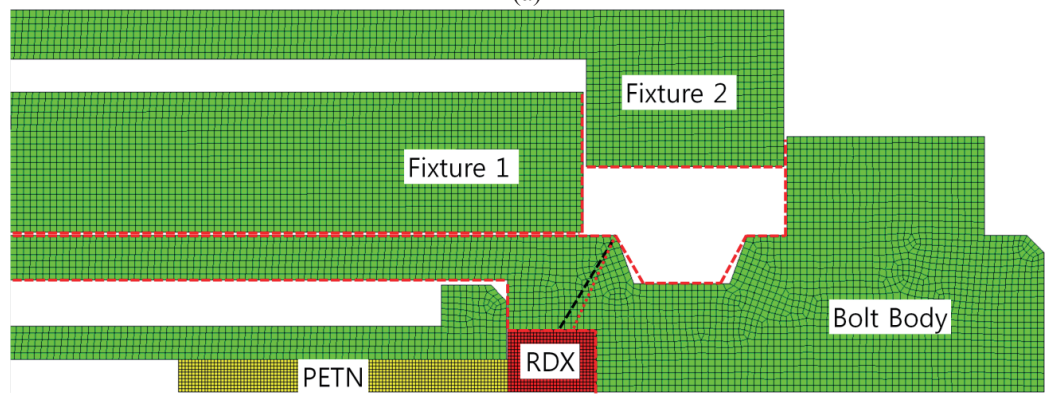

(b)

Fig. 9. Mesh with dashed reference outline for different ridge angle (a) Increased ridge angle model; (b) Decreased ridge angle model. 
waves. In the left-shifted ridge-position model, the distance from the RDX to the free surface of the ridge is reduced, which implies that the reflected release waves encounter trailing shock waves that are not sufficiently dissipated. Therefore, the tensile stress induced by the superposition of release waves is inadequate for failure,

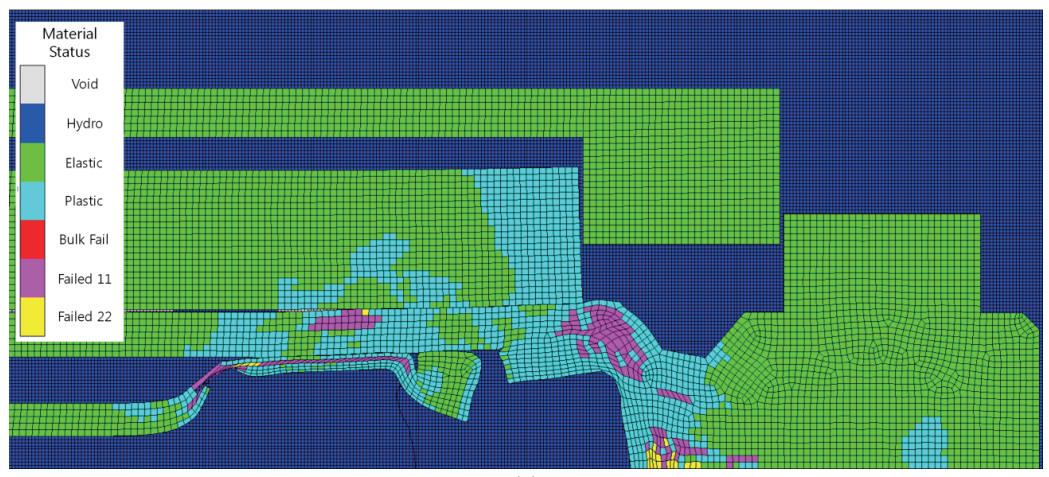

(a)

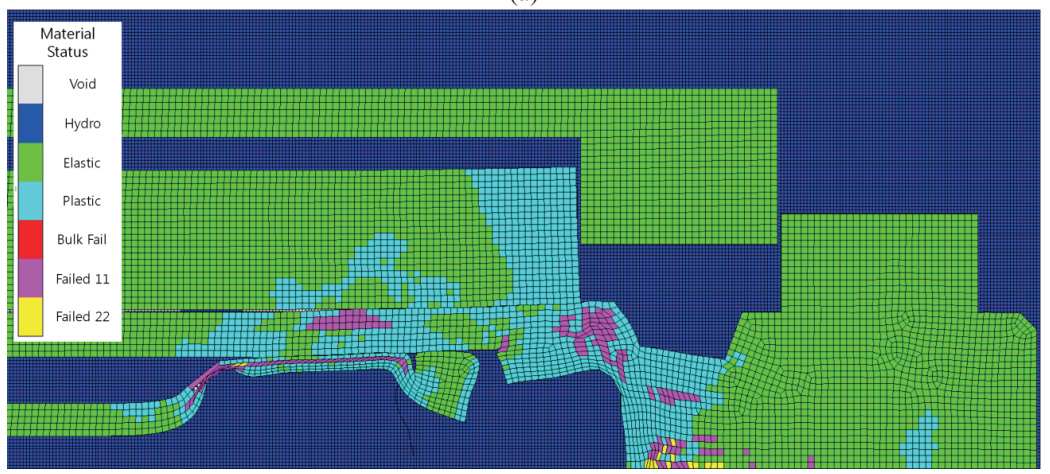

(b)

Fig. 10. Material statuses at $5.0 \mu$ s for different ridge angle (a) Increased ridge angle model; (b) Decreased ridge angle model.

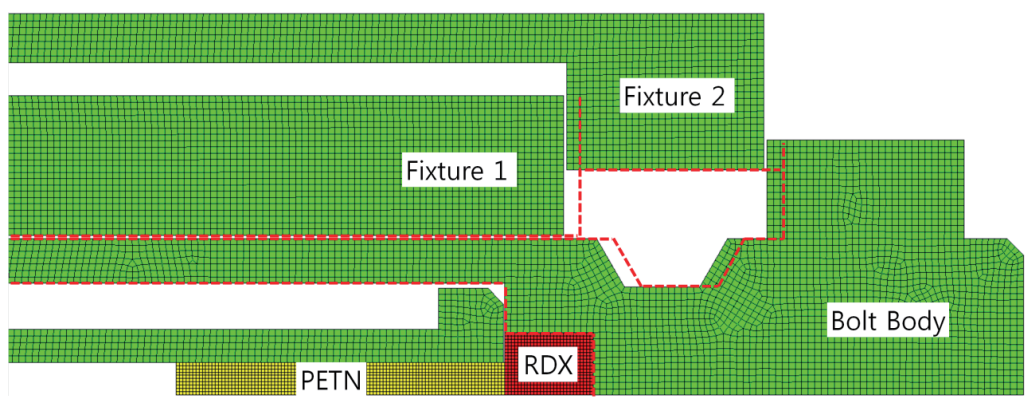

(a)

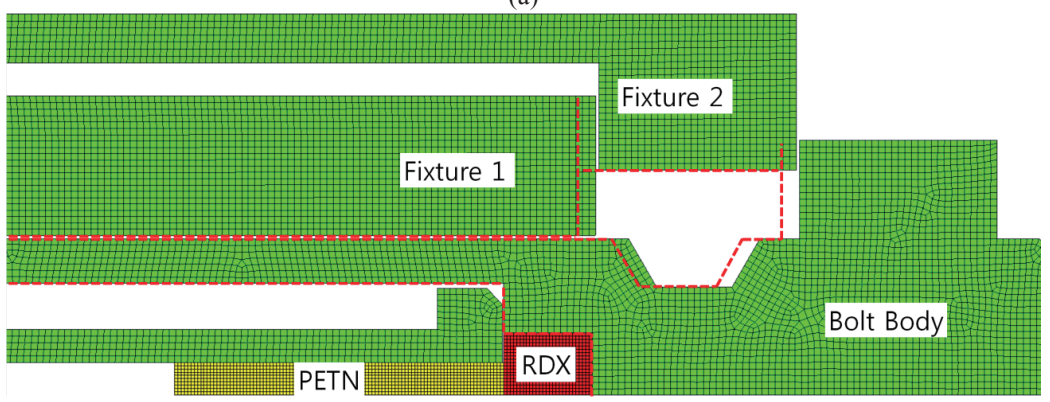

(b)

Fig. 11. Mesh with dashed reference outline for different ridge position (a) Left-shifted ridge-position model; (b) Right-shifted ridge-position model. 
which implies lower separation reliability. On the other hand, in the right-shifted ridge-position model, the distance from the RDX to the free surface of the ridge is increased, which implies that the reflected release waves encounter trailing shock waves that are already dissipated. Therefore, the tensile stress induced by the superposition of release waves is greater than that for the reference model. However, the right-shifted ridgeposition model has a larger volume around the ridge of the explosive bolt; and more elements (i.e., a larger portion of the bolt) must fail to induce bolt separation. Due to both effects, the separation reliability will be similar to the reference model.

\subsection{Summary of parametric study and design im- provements}

Based on this parametric study, practical design improvements are suggested for the reference explosive bolts. When the explosive weights of the high explosives are increased, the ridge angle is increased; and the separation reliability is increased. Therefore, with an increased ridge angle, the explosive weight can be reduced so that the side effects, such as pyroshock, are reduced even as the separation reliability similar to that of the reference model is maintained.

To determine the separation reliability of the increased

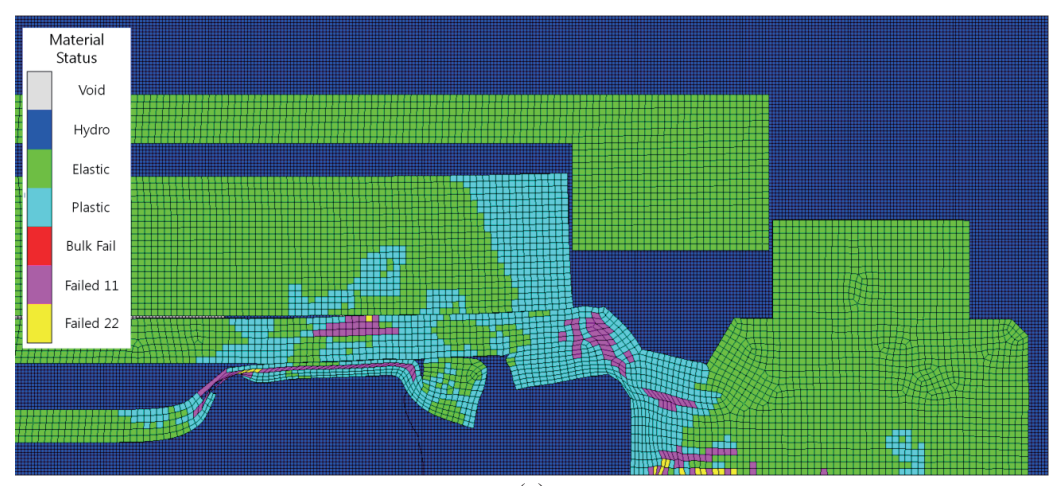

(a)

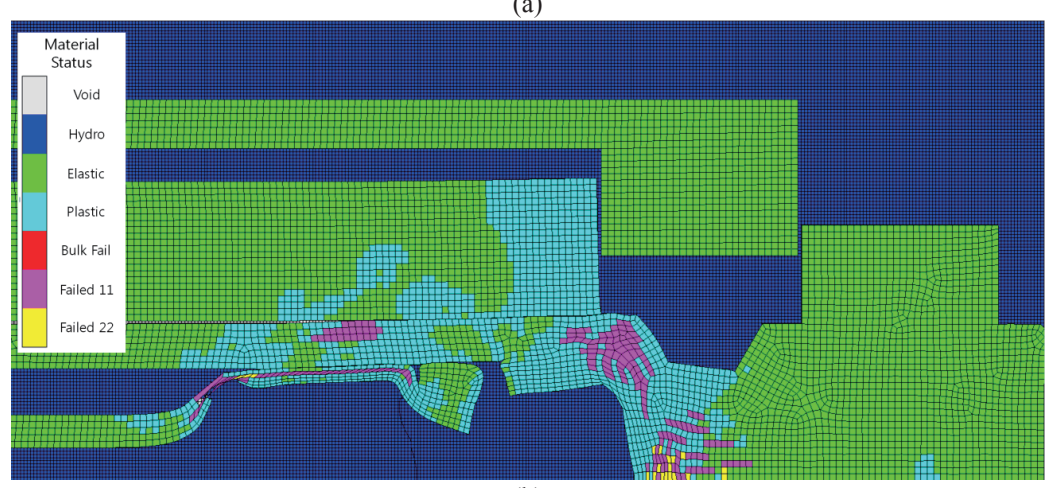

(b)

Fig. 12. Material statuses at 5.0 us for different ridge angle (a) Left-shifted ridge-position model; (b) Right-shifted ridge-position model.

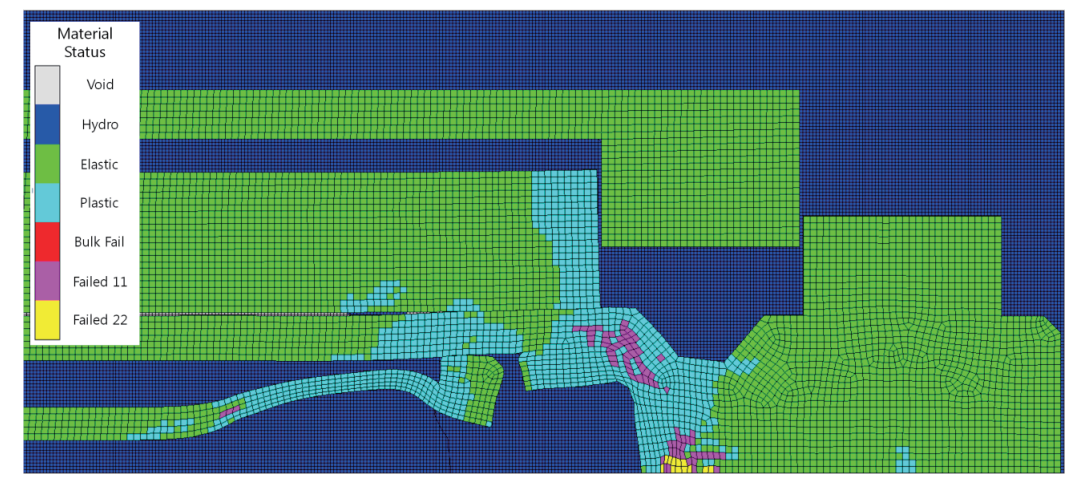

Fig. 13. Material statuses at $5.0 \mu$ s of increased ridge angle with decreased explosive weight model. 
ridge angle with decreased explosive weight model, the material status at $5.0 \mu$ s is shown in Fig. 13. Comparison between the results for the decreased explosive weight model (Fig. 8(c)), and the increased ridge angle with decreased explosive weight model (Fig. 13) shows that the separation reliability is slightly increased due to the effect of ridge angle increment. However, the separation reliability is lower than the reference model (Fig. 7(c)). From this observation, it is easily inferred that the separation reliability are mainly influenced by the explosive weight, rather than by the slight modification of ridge shape. However, the parametric study with design parameters of explosive bolts clearly shows that the separation reliability of ridge-cut explosive bolts are affected by the slight changes in ridge angle and position.

\section{Conclusion}

This study established the separation behavior analysis environments for ridge-cut explosive bolts, based on the ridge-cut mechanism. By using this analysis scheme, a parametric study of ridge-cut explosive bolts was carried out. Some design parameters were chosen that might affect the separation reliability, and the behavior analysis was carried out for several designs. Based on this parametric study, practical design improvements were suggested for the reference explosive bolts. When the explosive weights of the high explosives were increased, followed by an increase in the ridge angle the separation reliability was increased. Especially, the separation reliability was mainly influenced by the explosive weight, rather than by the slight modification of ridge shape.

\section{Acknowledgement}

This work was supported by the LIG Nexl under the contract YD11-3242.

\section{References}

[1] Brauer, K. O., Handbook of pyrotechnics, Chemical Publishing Co., New York, 1974.

[2] Bement, L. J. and Schimmel, M. L., A Manual for Pyrotechnic Design, Development and Qualification, NASA, NASA Technical Memorandum 110172, 1995.

[3] Kafadar, C. B., "The Mathematical Foundation for the "Ridge-Cut" Technique Used in Explosive Bolts", Annual
Meeting of the Pyrotechnics and Explosives Applications Section on the American Defense Preparedness Association, 1983.

[4] Balden, V. H. and Nurick, G. N., "Numerical simulation of the post-failure motion of steel plates subjected to blast loading", International Journal of Impact Engineering, Vol. 32, 2005, pp. 14-34.

[5] Bonorchis, D. and Nurick, G. N., "The influence of boundary conditions on the loading of rectangular plates subjected to localised blast loading - Importance in numerical simulations", International Journal of Impact Engineering, Vol. 36, No. 1, 2009, pp. 40-52.

[6] Bonorchis, D. and Nurick, G. N., "The analysis and simulation of welded stiffener plates subjected to localised blast loading", International Journal of Impact Engineering, Vol. 37, No. 3, 2010, pp. 260-273.

[7] Katayama, M. and Kibe, S., "Numerical study of the conical shaped charge for space debris impact", International Journal of Impact Engineering, Vol. 26, 2001, pp. 357-368.

[8] Katayama, M., Itoh, M., Tamura, S., Beppu, M. and Ohno, T., "Numerical analysis method for the RC and geological structures subjected to extreme loading by energetic materials", International Journal of Impact Engineering, Vol. 34, No. 9, 2007, pp. 1546-1561.

[9] Trelat, S., Sochet, I., Autrusson, B., Cheval, K. and Loiseau, O., "Impact of a shock wave on a structure on explosion at altitude", Journal of Loss Prevention in the Process Industries, Vol. 20, 2007, pp. 509-516.

[10] Trelat, S., Sochet, I., Autrusson, B., Loiseau, O. and Cheval, K., "Strong explosion near a parallelepipedic structure", Shock Waves, Vol. 16, 2007, pp. 349-357.

[11] Lee, J., Han, J.-H., Lee, Y. and Lee, H., "Separation characteristics study of ridge-cut explosive bolts", Aerospace Science and Technology, Vol. 39, 2014, pp. 153-168.

[12] Lee, Y. J., The Study of Development of Ridge-Cut Explosive Bolt (I), Agency for Defense Development, 2000.

[13] Lee, Y. J., The Study of Devlopment of Ridge-Cut Explosive Bolt (II), Agency for Defense Development, 2001.

[14] Lee, E. L., Hornig, H. C. and Kury, J. W., Adiabatic Expansion of High Explosive Detonation Products, Lawrence Radiation Laboratory, UCRL-50422, 1968.

[15] Zukas, J. A. and Walters, W. P., Explosive effects and applications, Springer, New York, 1998.

[16] Meyers, M. A., Dynamic behavior of materials, Wiley, New York, 1994.

[17] Cooper, P. W., Explosives engineering, VCH, New York, N.Y., 1996.

[18] Zukas, J. A., Introduction to hydrocodes, Elsevier, Amsterdam, 2004. 
Juho Lee A Parametric Study of Ridge-cut Explosive Bolts using Hydrocodes

[19] AK_Steel_Corporation, Product Data Bulletin : 17-4PH Stainless Steel, AK Steel Corporation, http:// www.aksteel.com/pdf/markets_products/stainless/ precipitation/17-4_PH_Stainless_Steel_PDB_201404.pdf, 2014.

[20] Weirick, L. J., Plane Shock Generator Explosive Lens: Shock Characterization of 4340 and PH13-8Mo Steels,
C360 Brass and PZT 65/35 Ferro-Electric Ceramic, Sandia National Laboratories, SAND93-3919, 1994.

[21] Johnson, G. R. and Cook, W. H., "A constitutive model and data for metals subjected to large strains, high strain rates and high temperatures", Proceedings of the Seventh International Symposium on Ballistics, The Hague, Netherlands, 1983. 